\title{
LTH 1124
}

\section{$F_{4}$ symmetric $\phi^{3}$ theory at four loops}

\author{
J.A. Gracey, \\ Theoretical Physics Division, \\ Department of Mathematical Sciences, \\ University of Liverpool, \\ P.O. Box 147, \\ Liverpool, \\ L69 3BX, \\ United Kingdom.
}

\begin{abstract}
The renormalization group functions for six dimensional scalar $\phi^{3}$ theory with an $F_{4}$ symmetry are provided at four loops in the modified minimal subtraction $(\overline{\mathrm{MS}})$ scheme. Aside from the anomalous dimension of $\phi$ and the $\beta$-function this includes the mass operator and a $\phi^{2}$-type operator. The anomalous dimension of the latter is computed explicitly at four loops for the $\mathbf{2 6}$ and $\mathbf{3 2 4}$ representations of $F_{4}$. The $\epsilon$ expansion of all the related critical exponents are determined to $O\left(\epsilon^{4}\right)$. For instance the value for $\Delta_{\phi}$ agrees with recent conformal bootstrap estimates in 5 and 5.95 dimensions. The renormalization group functions are also provided at four loops for the group $E_{6}$.
\end{abstract}




\section{Introduction.}

The mid 1980's saw a revolution in our understanding of two dimensional field theories due to the development and classification of conformal field theories, [1]. The extension beyond strictly two dimensions has not been as straightforward mainly due to the different structure of the underlying conformal group in two dimensions and $d>2$ where $d$ is the spacetime dimension. One recent development which is very promising is the so-called conformal bootstrap programme, [2, 3, 4, 5], which extended original ideas on higher dimensional conformal theories, [6, 17, 8, 9, 10, 11, 12]. Based on the earlier work of [2], the conformal bootstrap has led to a new way of estimating critical exponents in field theories in $d>2$. One primary example of the bootstrap success is in the three dimensional Ising model, [4], where estimates of exponents are competitive with other approaches such as strong coupling expansions, high temperature expansion and the $\epsilon$-expansion derived from perturbative renormalization group functions. A comprehensive review and summary of the results from these methods is given in [13]. A topic which has subsequently been part of this development is the study of scalar field theories at the Wilson-Fisher fixed point in dimensions greater than four. In [14, 15] it was demonstrated that $O(N) \phi^{4}$ theory could be extended into the $4<d<6$ range of spacetime dimensions and was in the same universality class as the $O(N)$ scalar $\phi^{3}$ theory which is perturbatively renormalizable in six dimensions. As well as the application of exact and functional renormalization group methods to this $O(N)$ theory conformal bootstrap studies also ensued with the main focus being the five dimensional theory, [16, 17, 18, 19, 20, 21, 22]. For instance, there has been a debate as to where the boundary of the conformal window, akin to that determined by the BanksZaks fixed point in gauge theories, [23], actually is. Bootstrap and functional renormalization group methods have yet to arrive at a consensus for even the ballpark area for the conformal window boundary $N_{\mathrm{cr}},[16,17,18,19,20,21,22$. Some bootstrap approaches find a low value for $N_{\mathrm{cr}}$ but others suggest a value in keeping with the estimates from the $\epsilon$ expansion at four loops, [14, 15, 24], which is in the neighbourhood of $N=400$. For instance, this value is not inconsistent with the mixed correlator bootstrap estimate of [22]. While such a discrepancy between different methods for $N_{\mathrm{cr}}$ has yet to be resolved what is not in question is that critical exponent estimates are in broad agreement. This is reassuring as ultimately if all methods had access to tools to refine their computations then they ought to agree precisely.

While the bootstrap debate to a degree has centred on quantum field theories with a classical Lie group symmetry, a recent study has concentrated on the exceptional group $F_{4}$, [25], as well as a brief look at the case of $E_{6}$ symmetry. This was partly to complement the study of the $d=6-2 \epsilon$ dimensional infrared stable fixed point with an $O(N)$ symmetry which can access the five dimensional theory. Clearly the issue of a conformal window is absent in the $F_{4}$ context in the sense that there is no range of a group parameter for which there is a Banks-Zaks type fixed point. However, [25] also provided another forum to explore the conformal bootstrap technology. Indeed in [25] the renormalization group functions for the six dimensional cubic $F_{4}$ symmetric field theory were determined to three loops in the modified minimal subtraction $(\overline{\mathrm{MS}})$ scheme. These were derived from the earlier results of [26, 27, 24]. One interesting outcome of [25] was the estimate for the field anomalous dimension in $d=5.95$ dimensions which was in precise agreement with that of the three loop $\epsilon$ expansion computed in perturbation theory. The study in $d=5$ dimensions was less clear in that the three loop estimate appeared to be out of line with that from the conformal bootstrap. While it was suggested this was due to non-perturbative effects, [25], one way to clarify this would be to extend the three loop $F_{4}$ perturbative renormalization group functions to four loops. This is the purpose of this article. We will determine the $\beta$-function and field and mass anomalous dimensions to four loops in the $\overline{\mathrm{MS}}$ scheme. However, as the bootstrap study in [25] involved other $\phi^{2}$-type operators we 
will compute their anomalous dimensions to four loops as well. Their one loop terms were given in [25. These extra operators are variants of the mass operator but in the $\mathbf{2 6}$ and $\mathbf{3 2 4}$ representations of $F_{4}$. They are required in the application of the operator product expansion decomposition of the product of fields into conformal primary operators in order to set up equations which the bootstrap technology solves. It turns out that having the critical exponents associated with these operators to the same level of accuracy gives insight into the interpretation of the estimate for the $d=5$ dimensional exponent for the field as well as the $\phi^{2}$-type operators. Indeed there is a suggestion that perturbative results from the underlying renormalization group functions could be used in future bootstrap studies as an aid or guide. For instance, in the $F_{4}$ case the exponent estimates derived from the $\epsilon$ expansion determine the order the operators appear in the spectrum in relation to increasing value. In addition we will provide the same renormalization group functions to four loops for other Lie groups in the family with underlying $F_{4}$ symmetry as well as the exceptional group $E_{6}$.

The article is organized as follows. We briefly recap the key aspects of the cubic scalar field theory in six dimensions upon which are computations are based in section 2 . This includes the definition of the $\phi^{2}$-type operators and an outline of the method we used to efficiently determine their four loop anomalous dimensions. To achieve this we need to use properties of the $F_{4}$ Lie algebra in order to evaluate the group factors associated with each Feynman graph. This is discussed in section 3 prior to the presentation of the renormalization group functions in section 4. Estimates for the critical exponents are also given there for the two specific dimensions of interest. Section 5 is devoted to the same analysis for the group $E_{6}$ while we provide conclusions in section 6. An appendix records the various critical exponents for the family of groups with related $F_{4}$ symmetry.

\section{Background.}

We begin by briefly recalling the necessary properties of the cubic scalar field theory in six dimensions. Our massless Lagrangian is

$$
L=\frac{1}{2}\left(\partial_{\mu} \phi^{i}\right)^{2}+\frac{g}{6} d^{i j k} \phi^{i} \phi^{j} \phi^{k}
$$

where $d^{i j k}$ is totally symmetric and the group indices have the range $1 \leq i \leq N$. We use the same coupling constant conventions as [24]. (As an aside we note the work of [28] where the exponent $\eta$ of the single field $\phi^{3}$ theory was computed directly using conformal bootstrap methods of [29] and can be regarded as a check on the results of [24].) The mass operator

$$
\mathcal{O}=\frac{1}{2} \phi^{i} \phi^{i}
$$

has been omitted as the new aspect of the renormalization of (2.1) here is that we will consider a set of $\phi^{2}$-type operators in different group representations and compute their anomalous dimensions to four loops. The anomalous dimension of one element of this set of operators is equivalent to that of $\mathcal{O}$ which is already known to four loops. To be more specific in order to build the various operators in a representation of $F_{4}$ there are five independent combinations of products of the group tensors $\delta^{i j}$ and $d^{i j k}$ with four free indices. These are $\delta^{i j} \delta^{k l}, \delta^{i k} \delta^{j l}, \delta^{i l} \delta^{j k}$, $d^{i k p} d^{j l p}$ and $d^{i l p} d^{j k p}$. The additional product of $d^{i j p} d^{k l p}$ is not independent due to the $F_{4} 4$-term relation. The various linear combinations of these tensors which correspond to projectors onto the $F_{4}$ representations were given in [30]. These lead to the set of rank $2 \phi^{2}$-type operators

$$
\mathcal{O}_{i j}^{(R)}=\frac{1}{2} \mathcal{P}_{i j k l}^{(R)} \phi^{k} \phi^{l}
$$


where

$$
\begin{aligned}
\mathcal{P}_{i j k l}^{(1)} & =\frac{1}{N} \delta_{i j} \delta_{k l}, \quad \mathcal{P}_{i j k l}^{(26)}=d_{i j p} d_{k l p} \\
\mathcal{P}_{i j k l}^{(324)} & =\frac{T_{2}}{2}\left[\delta_{i k} \delta_{j l}+\delta_{i l} \delta_{j k}-\frac{2}{N} \delta_{i j} \delta_{k l}\right]-d_{i j p} d_{k l p} \\
\mathcal{P}_{i j k l}^{(52)} & =\frac{8}{[N+10]}\left[\frac{T_{2}}{2}\left[\delta_{i l} \delta_{j k}-\delta_{i k} \delta_{j l}\right]+\frac{[N+2]}{8}\left[d_{i l p} d_{j k p}-d_{i k p} d_{j l p}\right]\right] \\
\mathcal{P}_{i j k l}^{(273)} & =\frac{[N+2]}{[N+10]}\left[\frac{T_{2}}{2}\left[\delta_{i l} \delta_{j k}-\delta_{i k} \delta_{j l}\right]-d_{i l p} d_{j k p}+d_{i k p} d_{j l p}\right]
\end{aligned}
$$

are the projectors in $F_{4}$, [30, 25]. Consequently the anomalous dimension of $\mathcal{O}$ and $\mathcal{O}_{i j}^{(1)}$ are equivalent. Also the anomalous dimensions for the $\mathbf{5 2}$ and $\mathbf{2 7 3}$ representations are immediately zero since the operator $\frac{1}{2} \phi^{k} \phi^{l}$ is symmetric whereas the respective projectors are antisymmetric in the indices $k$ and $l$. So our focus here will be on establishing the four loop anomalous dimensions of the symmetric $\mathbf{2 6}$ and $\mathbf{3 2 4}$ representations of $F_{4}$.

To determine the four loop anomalous dimensions of these operators we follow the same method as outlined in [24]. First, to renormalize an operator which does not mix it is inserted into the Green's function $\left\langle\phi^{i}(p) \mathcal{O}_{k l}^{(R)}(q) \phi^{j}(-p-q)\right\rangle$ where the external momenta $p$ and $q$ flow in through the external legs. Then evaluating the constituent Feynman graphs the renormalization constant for the operator is deduced from the poles in the regulator. In this paper we have used dimensional regularization in $d=6-2 \epsilon$ where $\epsilon$ is the regularizing parameter. Moreover we will use the modified minimal subtraction $(\overline{\mathrm{MS}})$ scheme to define the renormalization constants. While this outlines the essence of the standard operator renormalization procedure for the renormalization of the $\phi^{2}$-type operators defined from (2.1) there are several technical shortcuts which allow us to extract the four loop renormalization constants. As the operators do not involve derivatives then the insertion in the Green's function can be at zero momentum. If one was considering the renormalization of the mass operator in four dimensions then this nullification would be problematic. This is because the Feynman integrals would contain $1 /\left(k^{2}\right)^{2}$, where $k$ is a loop momentum, which is infrared divergent. In six dimensions, however, such a double pole propagator in an integral is infrared safe. So inserting the $\phi^{2}$-type operators at zero momentum will not corrupt the emergent operator renormalization constant with infrared divergences. Therefore we have relegated the exercise of renormalizing $\mathcal{O}_{i j}^{(R)}$ in effect to one of evaluating a 2-point function. As noted in [24] this could involve 540 Feynman diagrams to determine, for instance, for the nullified 3-point function. However, for the renormalization of $\mathcal{O}$ the Green's function $\left\langle\phi^{i}(p) \mathcal{O}(0) \phi^{j}(-p)\right\rangle$ was generated from the graphs of the $\phi^{i}$ 2-point function and we followed the same process here. For each graph of the $\phi^{i} 2$-point function one applies the map

$$
\frac{\delta_{i j}}{k^{2}} \mapsto \frac{\delta_{i j}}{k^{2}}+\frac{m^{2}}{2\left(k^{2}\right)^{2}}\left[\mathcal{P}_{i j k_{e} l_{e}}^{(R)}+\mathcal{P}_{i j l_{e} k_{e}}^{(R)}\right]
$$

to each propagator where we have not made any assumptions on the symmetry properties of the projection tensor. The particular combination which appears derives from the Feynman rule for $\mathcal{O}_{i j}^{(R)}$. The quantity $m$ is not a mass as such but a counting parameter. After the substitution has been made one truncates the graphs by retaining terms up to and including $O\left(\mathrm{~m}^{2}\right)$ only. Terms higher in $m^{2}$ would correspond to more than one insertion of the operator and correspond to a Green's function we are not interested in. The indices $k_{e}$ and $l_{e}$ are those associated with the external indices of the operator insertion. The advantage of using this technique to generate the particular Green's function is that it is straightforward to implement within our automatic Feynman diagram calculation. The graphs for the $\phi^{i}$ 2-point function are generated with the 
package QGRAF, [31, and converted into the syntax of the symbolic manipulation language we use which is FORM and its multithreaded version TFORM, [32, 33. As the computation we performed to determine the anomalous dimensions of the $\mathbf{2 6}$ and $\mathbf{3 2 4}$ representations of $\mathcal{O}^{(R)}$ used the same programmes as that for $\mathcal{O}$ we refer the interested reader to 24 for the technical details where the use of the Laporta algorithm, [34, and its implementation in REDUZE, [35, 36], is discussed in depth. The only major difference is that we have had to develop a Form module to handle the group theory associated with the $F_{4}$ tensor $d^{i j k}$.

\section{$3 \quad F_{4}$ group theory.}

We devote this section to the mechanics of finding the values for the group invariants which appear in the $\phi^{3}$ theory renormalization to four loops inclusive. These were defined in [26, 27] to three loops and the four loop ones were introduced in [24]. We use the notation introduced in the latter and for completeness we note that they are

$$
\begin{aligned}
& d^{i i_{1} i_{2}} d^{j i_{1} i_{2}}=T_{2} \delta^{i j}, d^{i i_{1} i_{2}} d^{j i_{1} i_{3}} d^{k i_{2} i_{3}}=T_{3} d^{i j k}, d^{i i_{1} i_{2}} d^{j i_{3} i_{4}} d^{k i_{5} i_{6}} d^{i_{1} i_{3} i_{5}} d^{i_{2} i_{4} i_{6}}=T_{5} d^{i j k} \\
& d^{i i_{1} i_{2}} d^{j i_{3} i_{4}} d^{k i_{5} i_{6}} d^{i_{1} i_{3} i_{7}} d^{i_{2} i_{5} i_{8}} d^{i_{4} i_{6} i_{9}} d^{i_{7} i_{8} i_{9}}=T_{71} d^{i j k} \\
& d^{i i_{1} i_{2}} d^{j i_{3} i_{4}} d^{k i_{5} i_{6}} d^{i_{1} i_{3} i_{7}} d^{i_{2} i_{5} i_{8}} d^{i_{4} i_{8} i_{9}} d^{i_{6} i_{7} i_{9}}=T_{72} d^{i j k} \\
& d^{i i_{1} i_{2}} d^{j i_{3} i_{4}} d^{k i_{5} i_{12}} d^{i_{1} i_{5} i_{6}} d^{i_{2} i_{7} i_{8}} d^{i_{3} i_{9} i_{12}} d^{i_{4} i_{7} i_{10}} d^{i_{6} i_{8} i_{11}} d^{i_{9} i_{10} i_{11}}=T_{91} d^{i j k} \\
& d^{i i_{1} i_{2}} d^{j i_{3} i_{4}} d^{k i_{11} i_{12}} d^{i_{1} i_{5} i_{6}} d^{i_{2} i_{7} i_{8}} d^{i_{3} i_{5} i_{9}} d^{i_{4} i_{7} i_{10}} d^{i_{6} i_{8} i_{11}} d^{i_{9} i_{10} i_{12}}=T_{92} d^{i j k} \\
& d^{i i_{1} i_{2}} d^{j i_{3} i_{4}} d^{k i_{6} i_{12}} d^{i_{1} i_{5} i_{6}} d^{i_{2} i_{7} i_{8}} d^{i_{3} i_{5} i_{9}} d^{i_{4} i_{7} i_{10}} d^{i_{8} i_{11} i_{12}} d^{i_{9} i_{10} i_{11}}=T_{93} d^{i j k} \\
& d^{i i_{1} i_{2}} d^{j i_{3} i_{4}} d^{k i_{5} i_{12}} d^{i_{1} i_{5} i_{6}} d^{i_{2} i_{7} i_{8}} d^{i_{3} i_{9} i_{12}} d^{i_{4} i_{10} i_{11}} d^{i_{6} i_{7} i_{10}} d^{i_{8} i_{9} i_{11}}=T_{94} d^{i j k} \\
& d^{i i_{1} i_{2}} d^{j i_{3} i_{4}} d^{k i_{8} i_{12}} d^{i_{1} i_{5} i_{6}} d^{i_{2} i_{7} i_{8}} d^{i_{3} i_{5} i_{9}} d^{i_{4} i_{10} i_{11}} d^{i_{6} i_{7} i_{10}} d^{i_{9} i_{11} i_{12}}=T_{95} d^{i j k} \\
& d^{i i_{1} i_{2}} d^{j i_{3} i_{4}} d^{k i_{11} i_{12}} d^{i_{1} i_{3} i_{5}} d^{i_{2} i_{6} i_{7}} d^{i_{4} i_{6} i_{8}} d^{i_{5} i_{9} i_{10}} d^{i_{7} i_{9} i_{11}} d^{i_{8} i_{10} i_{12}}=T_{96} d^{i j k} \\
& d^{i i_{1} i_{2}} d^{j i_{3} i_{4}} d^{k i_{5} i_{12}} d^{i_{1} i_{5} i_{6}} d^{i_{2} i_{7} i_{8}} d^{i_{3} i_{9} i_{12}} d^{i_{4} i_{7} i_{10}} d^{i_{6} i_{10} i_{11}} d^{i_{8} i_{9} i_{11}}=T_{97} d^{i j k} \\
& d^{i i_{1} i_{2}} d^{j i_{3} i_{4}} d^{k i_{6} i_{12}} d^{i_{1} i_{5} i_{6}} d^{i_{2} i_{7} i_{8}} d^{i_{3} i_{5} i_{9}} d^{i_{4} i_{7} i_{10}} d^{i_{8} i_{9} i_{11}} d^{i_{10} i_{11} i_{12}}=T_{98} d^{i j k} \\
& d^{i i_{1} i_{2}} d^{j i_{3} i_{4}} d^{k i_{11} i_{12}} d^{i_{1} i_{5} i_{6}} d^{i_{2} i_{7} i_{8}} d^{i_{3} i_{5} i_{9}} d^{i_{4} i_{7} i_{10}} d^{i_{6} i_{10} i_{11}} d^{i_{8} i_{9} i_{12}}=T_{99} d^{i j k} \text {. }
\end{aligned}
$$

In that article values were derived for certain groups and it transpired that several subsets of $T_{9 i}$ had the same values. We do not assume at the outset that the same feature arises for either $F_{4}$ or $E_{6}$ which is considered later.

As the first stage in the extraction of the four loop renormalization group functions for the $F_{4}$ symmetric case we recall basic properties of the tensor $d^{i j k}$ for this specific group. From [25, 30] the 4-term relation is

$$
d^{i j i_{1}} d^{k l i_{1}}+d^{i k i_{1}} d^{j i_{1}}+d^{i l i_{1}} d^{j k i_{1}}=\left[\delta^{i j} \delta^{k l}+\delta^{i k} \delta^{j l}+\delta^{i l} \delta^{j k}\right] \frac{2 T_{2}}{[N+2]}
$$

where we retain $T_{2}$ for completeness in contrast to [25]. From this we can derive an expression for the group theory value of a one loop box graph which we use extensively throughout. Although the same relation was given in [30] our derivation differs slightly from that given in [25, 30] but may be useful for constructing parallel expressions for the group theory associated with higher point one loop graphs. Contracting (3.2) with $d^{j p r} d^{k q r}$ produces

$$
\begin{aligned}
d^{i i_{1} i_{2}} d^{j i_{3} i_{2}} d^{k i_{1} i_{4}} d^{l i_{3} i_{4}}+d^{i i_{1} i_{2}} d^{j i_{3} i_{2}} d^{k i_{3} i_{4}} d^{l i_{1} i_{4}}= & {\left[d^{i k i_{1}} d^{j l i_{1}}+d^{i l i_{1}} d^{j k i_{1}}\right] \frac{2 T_{2}}{[N+2]} } \\
& +\delta^{i j} \delta^{k l} \frac{2 T_{2}^{2}}{[N+2]}
\end{aligned}
$$




$$
+d^{i j i_{1}} d^{k l i_{1}} \frac{[N-2] T_{2}}{2[N+2]}
$$

after relabelling. We have used $d^{i j j}=0$ which follows from contracting (3.2) with $d^{i j p}$. The two terms on the left hand side of (3.3) represent two permutations of the indices on a one loop box diagram. If we define the tensor

$$
B^{i j k l}=d^{i i_{1} i_{2}} d^{j i_{3} i_{2}} d^{k i_{1} i_{4}} d^{l i_{3} i_{4}}
$$

to denote the group theory associated with a one loop box integral and the right hand side of (3.3) formally by $C^{i j k l}$ then (3.3) becomes

$$
B^{i j k l}+B^{i j l k}=C^{i j k l} .
$$

By permuting the indices $j \rightarrow k, k \rightarrow l$ and $l \rightarrow j$ twice we obtain two other relations which are

$$
B^{i j l k}+B^{i k j l}=C^{i k l j}, B^{i k j l}+B^{i j k l}=C^{i l j k}
$$

where we have used $B^{i j k l}=B^{i l k j}$ which follows from the definition of $B^{i j k l}$. These three relations, (3.5) and (3.6), involve the three independent ways of labelling a one loop box topology and hence the equations can be represented by a $3 \times 3$ matrix where the entries are either 0 or 1 . Inverting this matrix then allows one to obtain the decomposition of a box topology into tensors involving fewer products of $d^{i j k}$ tensors which is, [25, 30],

$$
\begin{aligned}
d^{i i_{1} i_{3}} d^{j i_{2} i_{3}} d^{k i_{2} i_{4}} d^{l i_{1} i_{4}}= & {\left[\delta^{i j} \delta^{k l}-\delta^{i k} \delta^{j l}+\delta^{i l} \delta^{j k}\right] \frac{T_{2}^{2}}{[N+2]} } \\
& +\left[d^{i j i_{1}} d^{k l i_{1}}+d^{i l i_{1}} d^{j k i_{1}}\right] \frac{[N-2] T_{2}}{4[N+2]} \\
& -d^{i k i_{1}} d^{j l i_{1}} \frac{[N-10] T_{2}}{4[N+2]}
\end{aligned}
$$

where the right hand side is derived from $C^{i j k l}$ and its above permutations. The result is equivalent to that produced in [30] using a symmetrization and antisymmetrization method. Equipped with this has allowed us to evaluate all the group invariants of (3.1) aside from $T_{2}$. Its value will not be set to unity until later. We find

$$
\begin{aligned}
T_{3} & =-[N-2] \frac{T_{2}}{2[N+2]}, T_{5}=-\left[N^{2}-10 N-16\right] \frac{T_{2}^{2}}{2[N+2]^{2}} \\
T_{71} & =\left[N^{3}-3 N^{2}+80 N+100\right] \frac{T_{2}^{3}}{4[N+2]^{3}}, T_{72}=-N[N-2][N-10] \frac{T_{2}^{3}}{8[N+2]^{3}} \\
T_{91} & =[N-2]\left[N^{3}+18 N^{2}+204 N+152\right] \frac{T_{2}^{4}}{16[N+2]^{4}} \\
T_{92} & =T_{96}=T_{98}=-[N-2]\left[N^{3}-24 N^{2}+36 N+80\right] \frac{T_{2}^{4}}{8[N+2]^{4}} \\
T_{93} & =T_{95}=T_{97}=N[N-2]\left[N^{2}-24 N+12\right] \frac{T_{2}^{4}}{16[N+2]^{4}} \\
T_{94} & =-\left[N^{4}-14 N^{3}-12 N^{2}-616 N-672\right] \frac{T_{2}^{4}}{8[N+2]^{4}} \\
T_{99} & =[N-2]\left[N^{3}-27 N^{2}+54 N+72\right] \frac{T_{2}^{4}}{4[N+2]^{4}} .
\end{aligned}
$$


As a check we have reproduced the expressions for the invariants used in the three loop analysis of [25]. Those at four loops, $T_{9 n}$, are new. The only one where we could not directly reduce the invariant using (3.7) was $T_{99}$. This was because all the one loop subgraphs are pentagons and there are no boxes present. Instead we manufactured boxes by first applying the 4-term relation (3.2). An interesting feature emerges in (3.8). Setting $N=2$ in (3.8) the only non-zero invariants are $T_{5}, T_{71}$ and $T_{94}$ which all evaluate to unity. It transpires that for the exceptional group $E_{6}$, which we consider later, these are also the only non-zero invariants present in the renormalization group functions. Although their values will not be unity in that group. As we will be concentrating on the $N=26$ representation of $F_{4}$ we note that the specific values we used are

$$
\begin{aligned}
T_{2} & =1, T_{3}=-\frac{3}{7}, T_{5}=-\frac{25}{98}, T_{71}=\frac{277}{1372}, T_{72}=-\frac{39}{686} \\
T_{91} & =\frac{825}{9604}, T_{92}=T_{96}=T_{98}=-\frac{111}{9604}, T_{93}=T_{95}=T_{97}=\frac{39}{9604} \\
T_{94} & =-\frac{727}{19208}, T_{99}=\frac{75}{9604} .
\end{aligned}
$$

Here we have assumed $T_{2}=1$ like [25] for the purpose of comparing our renormalization group functions with that article.

\section{$4 \quad F_{4}$ renormalization group functions.}

Before examining the consequences of the four loop renormalization group functions for critical exponent estimates we first record the anomalous dimensions for the $\phi^{2}$-type operators in the $\mathbf{2 6}$ and $\mathbf{3 2 4}$ representations of $F_{4}$. These result from the method outlined in section 2 . For $\mathcal{O}_{i j}^{(26)}$ we have*

$$
\begin{aligned}
\gamma_{\mathcal{O}^{(26)}}(g)=- & \frac{T_{3}}{2} g^{2}+\left[-7 T_{2} T_{3}+18 T_{3}^{2}+12 T_{5}\right] \frac{g^{4}}{48} \\
+ & {\left[396 T_{2} T_{3}^{2}-119 T_{2}^{2} T_{3}+198 T_{2} T_{5}+486 T_{3}^{3}+2160 \zeta_{3} T_{3} T_{5}-2556 T_{3} T_{5}\right.} \\
& \left.-864 T_{71}-2592 \zeta_{3} T_{72}+864 T_{72}\right] \frac{g^{6}}{1728} \\
+ & {\left[1728 \zeta_{3} T_{2}^{3} T_{3}-36961 T_{2}^{3} T_{3}-15552 \zeta_{3} T_{2}^{2} T_{3}^{2}+188556 T_{2}^{2} T_{3}^{2}+48492 T_{2}^{2} T_{5}\right.} \\
& +23472 T_{2} T_{3}^{3}+964224 \zeta_{3} T_{2} T_{3} T_{5}-326592 \zeta_{4} T_{2} T_{3} T_{5}-820800 T_{2} T_{3} T_{5} \\
& -233280 T_{2} T_{71}-1073088 \zeta_{3} T_{2} T_{72}+419904 \zeta_{4} T_{2} T_{72}+233280 T_{2} T_{72} \\
& +933120 \zeta_{3} T_{3}^{4}-1502712 T_{3}^{4}-4157568 \zeta_{3} T_{3}^{2} T_{5}+933120 \zeta_{4} T_{3}^{2} T_{5} \\
& +3381696 T_{3}^{2} T_{5}+2737152 \zeta_{3} T_{3} T_{71}-7464960 \zeta_{5} T_{3} T_{71}+3877632 T_{3} T_{71} \\
& +3048192 \zeta_{3} T_{3} T_{72}-1119744 \zeta_{4} T_{3} T_{72}-3877632 T_{3} T_{72}-653184 \zeta_{3} T_{5}^{2} \\
& +660960 T_{5}^{2}+3732480 \zeta_{3} T_{91}-3732480 \zeta_{5} T_{91}+373248 T_{91}+3732480 \zeta_{3} T_{98} \\
& -3732480 \zeta_{5} T_{98}-746496 \zeta_{3} T_{99}+933120 \zeta_{5} T_{99}+1866240 \zeta_{3} T_{92} \\
& -1866240 \zeta_{5} T_{92}-4354560 \zeta_{3} T_{93}+6220800 \zeta_{5} T_{93}+1119744 \zeta_{3} T_{94} \\
& -373248 T_{94}-6096384 \zeta_{3} T_{95}+8709120 \zeta_{5} T_{95}+3732480 \zeta_{3} T_{96} \\
& \left.-3732480 \zeta_{5} T_{96}-2612736 \zeta_{3} T_{97}+3732480 \zeta_{5} T_{97}\right] \frac{g^{8}}{746496}+O\left(g^{10}\right)
\end{aligned}
$$

in the $\overline{\mathrm{MS}}$ scheme where $\zeta(z)$ is the Riemann zeta function. We have been able to determine this without reference to specific $F_{4}$ related group identities such as (3.2) and (3.7). In other words

${ }^{*}$ We have attached an electronic data file with the operator anomalous dimensions. 
to four loops the combination of $d^{i j k}$ tensors in each graph could be written in terms of one of the group invariants of (3.1). Therefore (4.1) can be used for the non- $F_{4}$ symmetric problems discussed in [24]. The situation for the remaining $\phi^{2}$-type operator is that we have had to use $F_{4}$ based identities as noted earlier. So the four loop anomalous dimension is not expressed in terms of the $T_{i}$ invariants of (3.1) and can only be used in the $F_{4}$ context. In the $\overline{\mathrm{MS}}$ scheme we found

$$
\begin{aligned}
\gamma_{\mathcal{O}^{(324)}}(g)=- & \frac{g^{2}}{[N+2]}+[5 N+22] \frac{g^{4}}{24[N+2]^{2}} \\
- & {\left[1728 \zeta_{3} N^{2}-1465 N^{2}-8640 \zeta_{3} N+13724 N+7316\right] \frac{g^{6}}{864[N+2]^{3}} } \\
+ & {\left[3290976 \zeta_{3} N^{3}+641520 \zeta_{4} N^{3}-5870880 \zeta_{5} N^{3}+1604411 N^{3}-48224160 \zeta_{3} N^{2}\right.} \\
& \quad-2426112 \zeta_{4} N^{2}+76515840 \zeta_{5} N^{2}-17175342 N^{2}+105473664 \zeta_{3} N \\
& +1353024 \zeta_{4} N-165939840 \zeta_{5} N+52790148 N+2595456 \zeta_{3}-3359232 \zeta_{4} \\
& \left.-31726080 \zeta_{5}+53133016\right] \frac{g^{8}}{373248[N+2]^{4}}+O\left(g^{10}\right) .
\end{aligned}
$$

The general expressions for $\gamma_{\phi}(g), \gamma_{\mathcal{O}}(g)$ and $\beta(g)$ were given in earlier in [24] in terms of the group invariants $T_{i}$ and we do not reproduce them here for $F_{4}$ as these were given in [25] at three loops. Instead we have evaluated them for the case when $N=26$ and together with (4.1) and (4.2) we have

$$
\begin{aligned}
\beta(g)= & \frac{1}{4}(d-6) g-\frac{19}{56} g^{3}-\frac{1997}{14112} g^{5}+\left[3301747-3383856 \zeta_{3}\right] \frac{g^{7}}{14224896} \\
& +\left[1259178385-596452464 \zeta_{3}+192879792 \zeta_{4}-926795520 \zeta_{5}\right] \frac{g^{9}}{1194891264} \\
& +O\left(g^{11}\right) \\
\gamma_{\phi}(g)= & -\frac{1}{12} g^{2}-\frac{149}{3024} g^{4}-\left[32400 \zeta_{3}+78731\right] \frac{g^{6}}{3048192} \\
& +\left[3121981-1958160 \zeta_{3}-256176 \zeta_{4}-1595520 \zeta_{5}\right] \frac{g^{8}}{28449792}+O\left(g^{10}\right) \\
\gamma_{\mathcal{O}}(g)= & -\frac{1}{2} g^{2}-\frac{79}{336} g^{4}-\left[16848 \zeta_{3}+34631\right] \frac{g^{6}}{84672} \\
& +\left[230779145-258668208 \zeta_{3}+21403440 \zeta_{4}-89579520 \zeta_{5}\right] \frac{g^{8}}{256048128} \\
& +O\left(g^{10}\right) \\
& \frac{3}{14} g^{2}+\frac{53}{784} g^{4}+\left[5481 \zeta_{3}-6689\right] \frac{g^{6}}{24696} \\
& +\left[236544192 \zeta_{3}-104509440 \zeta_{4}+413138880 \zeta_{5}-531246791\right] \frac{g^{8}}{597445632} \\
& +O\left(g^{10}\right) \\
\gamma_{\mathcal{O}^{(26)}}(g)= & \frac{1}{28} g^{2}+\frac{19}{2352} g^{4}+\left[78275-117936 \zeta_{3}\right] \frac{g^{6}}{2370816} \\
& +\left[218652912 \zeta_{3}+75524400 \zeta_{4}-436000320 \zeta_{5}+140736511\right] \frac{g^{8}}{1792336896} \\
& +O\left(g^{10}\right) \\
\gamma_{\mathcal{O}^{(324)}}(g)= & (4.3) \\
= &
\end{aligned}
$$

using (3.9). Numerically we have

$$
\beta(g)=-0.500000 \epsilon g-0.339286 g^{3}-0.141511 g^{5}-0.053838 g^{6}-0.175793 g^{8}
$$




$$
\begin{aligned}
& +O\left(g^{11}\right) \\
\gamma_{\phi}(g)= & -0.083333 g^{2}-0.049272 g^{4}-0.038606 g^{6}-0.040898 g^{8}+O\left(g^{10}\right) \\
\gamma_{\mathcal{O}}(g)= & -0.500000 g^{2}-0.235119 g^{4}-0.648187 g^{6}-0.585346 g^{8}+O\left(g^{10}\right) \\
\gamma_{\mathcal{O}^{(26)}}(g)= & 0.214286 g^{2}+0.067602 g^{4}-0.0040705 g^{6}+0.114445 g^{8}+O\left(g^{10}\right) \\
\gamma_{\mathcal{O}^{(32)}}(g)= & -0.035714 g^{2}+0.008078 g^{4}-0.026780 g^{6}+0.018529 g^{8}+O\left(g^{10}\right)
\end{aligned}
$$

where the four loop terms are roughly the same magnitude as the lower order ones. The three loop values for the first three renormalization group functions are in agreement with [25]. Clearly there is no sign of a Banks-Zaks fixed point to four loops with our coupling constant conventions. With the coupling constant conventions of [25] there are fixed points for the two and four loop $\beta$-functions but only complex solutions to $\beta(g)=0$ at three loops. So there is no robust BanksZaks fixed point which would in fact be an asymptotically safe solution if it had existed.

\begin{tabular}{|c||c|c|c|}
\hline$d$ & 2 loop & 3 loop & 4 loop \\
\hline 5 & 1.5731551 & 1.5613412 & 1.5639085 \\
5.95 & 1.9780535 & 1.9780512 & 1.9780512 \\
\hline
\end{tabular}

Table 1. $[0, l]$ Padé approximants for $F_{4}$ exponent $\Delta_{\phi}$ at $l$-loops.

Equipped with these renormalization group functions we can evaluate the $\epsilon$ expansion of the related critical exponents where the fixed point, $g_{c}$, is defined by $\beta\left(g_{c}\right)=0$. In order to compare with the results of [25] we use the notation of that article but define the exponents with respect to the conventions used here. We recall, [25],

$$
\begin{aligned}
\Delta_{\phi} & =\frac{1}{2} d-1+\gamma_{\phi}\left(g_{c}\right) \\
\Delta_{\mathcal{O}^{(R)}} & =d-2+2 \gamma_{\phi}\left(g_{c}\right)-2 \gamma_{\mathcal{O}^{(R)}}\left(g_{c}\right) \\
\Delta_{\phi^{3}} & =d+\beta^{\prime}\left(g_{c}\right)
\end{aligned}
$$

where $\gamma_{\mathcal{O}^{(1)}}\left(g_{c}\right) \equiv \gamma_{\mathcal{O}}\left(g_{c}\right)$. Solving for $g_{c}$ and evaluating these exponents we find

$$
\begin{aligned}
\Delta_{\phi} & =\frac{1}{2} d-1+0.122807 \epsilon-0.031524 \epsilon^{2}+0.042483 \epsilon^{3}+0.122722 \epsilon^{4}+O\left(\epsilon^{5}\right) \\
\Delta_{\mathcal{O}^{(1)}} & =d-2-1.228070 \epsilon+0.052388 \epsilon^{2}-3.414275 \epsilon^{3}-3.252345 \epsilon^{4}+O\left(\epsilon^{5}\right) \\
\Delta_{\mathcal{O}^{(26)}} & =d-2+1.122807 \epsilon-0.031524 \epsilon^{2}+0.042483 \epsilon^{3}+0.122722 \epsilon^{4}+O\left(\epsilon^{5}\right) \\
\Delta_{\mathcal{O}^{(324)}} & =d-2+0.140351 \epsilon-0.162835 \epsilon^{2}-0.172846 \epsilon^{3}-0.472810 \epsilon^{4}+O\left(\epsilon^{5}\right) \\
\Delta_{\phi^{3}} & =d+2 \epsilon-1.229301 \epsilon^{2}-0.132727 \epsilon^{3}-8.882515 \epsilon^{4}+O\left(\epsilon^{5}\right)
\end{aligned}
$$

where the terms to three loops of $\Delta_{\phi}, \Delta_{\mathcal{O}^{(1)}}$ and $\Delta_{\phi^{3}}$ are in agreement with [25]. Also the coefficients of $\Delta_{\phi}$ and $\Delta_{\mathcal{O}^{(26)}}$ are consistent with $\mathcal{O}^{(26)}$ being a conformal descendant of $\phi^{i}$. Surprisingly the four loop correction to $\Delta_{\phi^{3}}$ is large. One of the main features of [25] was the comparison of the exponent $\Delta_{\phi}$ with the value obtained from the conformal bootstrap analysis for dimensions $d=5$ and 5.95. In Tables 1 and 2 we have provided estimates for $\Delta_{\phi}$ using Padé approximants at successive loop orders in order to gauge convergence. In Table 1 we note the $[0, l]$ estimate at the $l$-loop order. The results for $d=5.95$ dimension appear to converge while those for $d=5$ have not converged as well but do appear to have settled to a value in the neighbourhood of 1.56. In compiling the Padé analysis what was apparent was that the estimates from the other $[p, q]$ approximants were not dissimilar to the $[0, l]$ ones. Therefore to improve estimates we calculated the average of all the approximants at each loop. These are presented in Table 2 and indicates a four loop value closer to 1.55 . 


\begin{tabular}{|c||c|c|c|}
\hline$d$ & 2 loop & 3 loop & 4 loop \\
\hline 5 & 1.5632667 & 1.5589076 & 1.5516367 \\
5.95 & 1.9780520 & 1.9780511 & 1.9780512 \\
\hline
\end{tabular}

Table 2. Average of Padé approximants at each loop order for $F_{4}$ exponent $\Delta_{\phi}$.

\begin{tabular}{|c|c||c|c|c|}
\hline$d$ & $R$ & 2 loop & 3 loop & 4 loop \\
\hline 5 & $\mathbf{2 6}$ & 3.5598518 & 3.5585128 & 3.5648212 \\
& $\mathbf{3 2 4}$ & 3.0848268 & 3.0317658 & 2.9986463 \\
\hline 5.95 & $\mathbf{2 6}$ & 3.9780513 & 3.9780511 & 3.9780512 \\
& $\mathbf{3 2 4}$ & 3.9534156 & 3.9534045 & 3.9534041 \\
\hline
\end{tabular}

Table 3. $[0, l]$ Padé approximants for $F_{4}$ exponent $\Delta_{\mathcal{O}^{(R)}}$ at $l$-loops in dimension $d$.

Given these estimates for $\Delta_{\phi}$ we have repeated the same Padé analysis for the $\phi^{2}$-type operators in the $\mathbf{2 6}$ and $\mathbf{3 2 4}$ representations as these are the only cases with non-zero critical exponents. The results are presented in Tables 3 and 4 where we gather the 5 and 5.95 dimension estimates in each table. The former Table has the values for the $[0, l]$ Padé approximants at $l$-loops and the latter has the average of the Padé's at each loop order. For 5.95 dimensions the exponents agree to at least four decimal places for each of the representations. In five dimensions the convergence is not as fast but again there appears to be a consistent value to two decimal places. In terms of comparing the exponent values in different representations in a particular dimension the operators are virtually degenerate in 5.95 dimensions. The discrepancy between them is around $0.5 \%$. As the spacetime dimension decreases this effective degeneracy is lifted. It transpires that the critical exponent for the $\mathbf{3 2 4}$ representation, $\Delta_{\mathcal{O}^{(324)}}$, is lower than that of the 26 representation, $\Delta_{\mathcal{O}^{(26)}}$. Moreover the former decreases more rapidly than the one for the 26 representation as the spacetime dimension decreases. A similar feature was evident in the analysis of $[25]$.

It is now instructive to compare our four loop estimates with the conformal bootstrap results of [25]. In that article plots were given of the allowed and excluded regions of the parameter space defined by $\Delta_{\phi}$ (x-axis) and what was termed $\Delta_{\mathbf{2 6}}^{2 \text { nd }}$ (y-axis) in the notation of [25]. In the bootstrap approach the location of a kink in the boundary of these two regions is the point where one can read off an estimate for $\Delta_{\phi}$ for example from the $x$-coordinate. First, the plot of 25 in 5.95 dimensions, for instance, has a well defined kink with an $x$-coordinate value of 1.978. This is in accord with the three loop $\epsilon$ series estimates provided in [25]. Our new four loop values in Tables 1 and 2 are not inconsistent with this. In addition what is apparent from the plot of [25] in this spacetime dimension is that the $y$-coordinate corresponds to a value fractionally shy of 4 . This is the estimate given in [25] for the quantity $\Delta_{\mathbf{2}}^{2 n d}$. This is not dissimilar to the estimates of both $\Delta_{\mathcal{O}^{(26)}}$ and $\Delta_{\mathcal{O}^{(324)}}$ shown in Tables 3 and 4 . Indeed our four loop estimates show a small change from the one loop estimates given in Table 1 of [25] for these exponents.

The situation for the conformal bootstrap analysis in five dimensions is different. While there is an allowed and excluded region in the corresponding plot of [25] there are no sharp kinks or boundaries. Instead there are what was termed weak kinks, [25]. From Figure 4 of [25] the kinks have slope changes at about 1.55 and 1.6 in the $x$-coordinate. Indeed the latter value is what was quoted for the bootstrap estimate for $\Delta_{\phi}$. However, from Table 2 the average four loop Padé estimate for $\Delta_{\phi}$ falls closer to the value of 1.55 rather than the quoted value of 1.6 in [25]. Indeed it was noted in [25] that this latter value was not in full agreement with the 
three loop perturbation theory used in [25]. Our four loop result shows that the series for $\Delta_{\phi}$ is not diverging as is apparent from Table 2. Put another way if one regards the lower kink of Figure 4 of [25] as the one to be used for estimating exponents then the perturbative result is not inconsistent with the bootstrap technology. To support this point of view one can examine the location of the first weak kink or lower knuckle of Figure 4 in [25] with respect to the $y$-axis. This is roughly at 3.1, 25]. Our estimates for $\Delta_{\mathcal{O}^{(324)}}$ are in the region of 3.01 which appears to be consistent with the $y$-axis value of Figure 4 in [25]. For the $\mathbf{2 6}$ representation we find an estimate for the $\Delta_{\mathcal{O}^{(26)}}$ exponent of around 3.55. This is lower than the upper knuckle of the five dimensional plot in 25] which appears closer to 3.9. However, the three curves presented in Figure 4 of [25] have not converged to the same accuracy as those in the neighbourhood of the lower knuckle in 5 dimensions or indeed that for 5.95 dimensions. Moreover in the latter spacetime dimension it is the lower corner of the plot of Figure 4 of [25] which gives the dimension of either $\phi^{2}$-type operator. That should also be the case in 5 dimensions in order to have a consistent point of view. What is interesting is that the conformal bootstrap analysis appears to give relatively accurate data on the exponent of the operator with the lowest value. However if one wishes to marry the information derived from perturbation theory here with the data from the 5 dimensional bootstrap analysis then one would have to regard the exponent estimate from the lower kink as corresponding to that of the $\phi^{2}$-type operator in the $\mathbf{3 2 4}$ representation.

\begin{tabular}{|c|c||c|c|c|}
\hline$d$ & $R$ & 2 loop & 3 loop & 4 loop \\
\hline 5 & $\mathbf{2 6}$ & 3.5566150 & 3.5578930 & 3.5509073 \\
& $\mathbf{3 2 4}$ & 3.0562147 & 3.0062392 & 3.0267278 \\
\hline 5.95 & $\mathbf{2 6}$ & 3.9780509 & 3.9780511 & 3.9780512 \\
& $\mathbf{3 2 4}$ & 3.9534112 & 3.9534043 & 3.9534041 \\
\hline
\end{tabular}

Table 4. Average of Padé approximants at each loop order for $F_{4}$ exponent $\Delta_{\mathcal{O}^{(R)}}$ at $l$-loops in dimension $d$.

\section{$5 E_{6}$.}

For the remaining part of our study of $\phi^{3}$ theory with exceptional symmetry we concentrate on the group $E_{6}$ where the fundamental representation is $\mathbf{2 7}$ and the adjoint is $\mathbf{7 8}$. As $E_{6}$ is a complex group then the Lagrangian for a cubic theory with $E_{6}$ symmetry involves fields $\phi^{i}$ and $\bar{\phi}^{i}$ and the tensors $d_{i j k}$ and $d^{i j k}$. We take the convention that the conjugate to $d^{i j k}$ is $d_{i j k}$ similar to [25]. Therefore the $E_{6}$ symmetric Lagrangian is

$$
L=\partial_{\mu} \bar{\phi}_{i} \partial^{\mu} \phi^{i}+\frac{g}{6}\left(d_{i j k} \phi^{i} \phi^{j} \phi^{k}+d^{i j k} \bar{\phi}_{i} \bar{\phi}_{j} \bar{\phi}_{k}\right)
$$

This is similar in structure to the cubic theory with $S U(3) \times S U(3)$ symmetry considered in [37, 38]. Moreover, the Feynman graphs generated from (5.1) share properties similar to those of the $S U(3) \times S U(3)$ theory. The main one is that there are no Feynman diagrams with subgraphs with an odd number of legs. So for instance there is no one loop triangle graph for the renormalization of the coupling constant. It is straightforward to establish this by realising that the two vertices of (5.1) are what is termed directed. Either all the arrows indicating the charge flow on each vertex line points to the interaction point itself or points away. Thus it is easy to see that in a one loop triangle graph the lines cannot be decorated with arrows which point to or from all the vertices. To reflect this aspect of the properties of the $E_{6}$ Lagrangian the indices of the tensor associated with the coupling constant are either raised or lowered, [25]. 
This convention will only be applied in this section. Moreover we will use upper and lower group indices on the fields themselves in keeping with the notion of distinguishing that there is a flow of charge in contrast to $F_{4}$.

To construct the four loop $E_{6}$ renormalization group functions we need to determine the values of the group invariants. The properties of the $E_{6}$ Lie algebra differ from those of $F_{4}$ but we will use the same algorithm as before to derive an identity for the one loop box akin to (3.7). We base our derivations on $E_{6}$ group properties derived in [39] which used the more mathematical articles [40, 41, 42, 43]. Further background to the structure and properties of $E_{6}$ can be found in [30, 44]. In [39] the convention for the product of tensors was

$$
d_{i k l} d^{j k l}=10 \delta_{i}{ }^{j}
$$

which imples $T_{2}=10$. From this and identities derived in [40, 41, 42, 43] it was shown in [39] that

$$
d_{i i_{1} i_{3}} d^{j i_{2} i_{3}} d_{k i_{2} i_{4}} d^{l i_{1} i_{4}}=5\left[\delta_{i}^{j} \delta_{k}^{l}+\delta_{i}{ }^{l} \delta_{k}^{j}\right]-4 d_{i k i_{1}} d^{j l i_{1}} .
$$

This is the $E_{6}$ equivalent of the one loop box topology of (3.7). Using this we have determined the values of all the invariants of (3.1). Before recording the values we note that for topologies where at least one of the one loop subgraphs has an odd number of external legs it is not possible to decorate the lines consistently in such a way that all the vertices have all arrows pointing in or out. In these cases the invariant is set to zero as such Feynman graphs would not be generated in the first place using, say, the QGRAF package, 31. It transpires that to four loops there are only three non-zero invariants aside from $T_{2}$. In summary the values are

$$
T_{2}=10, T_{5}=-30, T_{71}=220, T_{94}=-530
$$

after applying the box rule to (3.1).

It is a straightforward exercise to substitute these values in the expressions for the renormalization group functions which have been expressed in terms of $T_{i}$ to find

$$
\begin{aligned}
\beta(g)= & \frac{1}{4}(d-6) g-\frac{5}{4} g^{3}+\frac{265}{72} g^{5}+5\left[163183-19440 \zeta_{3}\right] \frac{g^{7}}{5184} \\
& +5\left[1044144 \zeta_{3}+32400 \zeta_{4}-633600 \zeta_{5}+591527\right] \frac{g^{9}}{3456}+O\left(g^{11}\right) \\
\gamma_{\phi}(g)= & -\frac{5}{6} g^{2}-\frac{275}{108} g^{4}+25\left[2699-3888 \zeta_{3}\right] \frac{g^{6}}{7776} \\
& +25\left[143119-9936 \zeta_{3}+6480 \zeta_{4}-126720 \zeta_{5}\right] \frac{g^{8}}{5184}+O\left(g^{10}\right) \\
\gamma_{\mathcal{O}}(g)= & -5 g^{2}-\frac{25}{12} g^{4}-\frac{9575}{108} g^{6} \\
& +25\left[3401136 \zeta_{3}-174960 \zeta_{4}-10264320 \zeta_{5}+13869707\right] \frac{g^{8}}{46656}+O\left(g^{10}\right) \\
\gamma_{\mathcal{O}(27)}(g)= & -\frac{15}{2} g^{4}-\frac{1155}{8} g^{6}+1055\left[49-432 \zeta_{3}\right] \frac{g^{8}}{288}+O\left(g^{10}\right)
\end{aligned}
$$

where there is no one loop contribution to the final renormalization group function. Numerically we have

$$
\begin{aligned}
\beta(g)= & -0.500000 \epsilon-1.250000 g^{3}+3.680556 g^{5}+134.852444 g^{7}+1771.871166 g^{9} \\
& +O\left(g^{11}\right) \\
\gamma_{\phi}(g)= & -0.833333 g^{2}-2.546296 g^{4}-6.348371 g^{6}+32.741763 g^{8}+O\left(g^{10}\right) \\
\gamma_{\mathcal{O}}(g)= & -5.000000 g^{2}-2.083333 g^{4}-88.657407 g^{6}+3818.021497 g^{8}+O\left(g^{10}\right) \\
\gamma_{\mathcal{O}^{(27)}}(g)= & -7.500000 g^{4}-144.375000 g^{6}-1722.758521 g^{8}+O\left(g^{10}\right) .
\end{aligned}
$$


In [25] it was noted that from the one loop $\beta$-function there was a stable unitary fixed point which we confirm here allowing for the different convention on the definition of the sign of our coupling constant in (2.1). Compared to the $F_{4} \beta$-function the coefficients of the $E_{6} \beta$-function increase significantly with the loop order. This can be traced, however, to the different values of $T_{2}$ which is 10 for $E_{6}$ instead of unity for $F_{4}$. If one rescaled $g^{2}$ by a factor of 10 then the coefficients of $\beta(g)$ would be comparable to those of $F_{4}$. In our coupling constant conventions the $E_{6}$ case like $F_{4}$ exhibits asymptotic freedom and to four loops has a Banks-Zaks fixed point. At two, three and four loops this is at $g^{2}=0.339623,0.083593$ and 0.063944 respectively. The latter values suggest convergence. At three loops there is a fixed point for negative coupling which is not present at two or four loops. If it had been present in those cases then $E_{6}$ could be a model with the property of asymptotic safety.

In advance of a conformal bootstrap analysis we can now provide the $\epsilon$ expansion of the related critical exponents at the Wilson-Fisher fixed point at four loops. These are

$$
\begin{aligned}
\Delta_{\phi} & =\frac{1}{2} d-1+0.333333 \epsilon-0.800000 \epsilon^{2}+8.044444 \epsilon^{3}-84.333501 \epsilon^{4}+O\left(\epsilon^{5}\right) \\
\Delta_{\mathcal{O}^{(1)}} & =d-2-3.333333 \epsilon+3.777778 \epsilon^{2}-76.971365 \epsilon^{3}+505.735425 \epsilon^{4}+O\left(\epsilon^{5}\right) \\
\Delta_{\mathcal{O}^{(27)}} & =d-2+1.333333 \epsilon-0.800000 \epsilon^{2}+8.044444 \epsilon^{3}-84.333501 \epsilon^{4}+O\left(\epsilon^{5}\right) \\
\Delta_{\phi^{3}} & =d+2 \epsilon+2.355556 \epsilon^{2}-74.593093 \epsilon^{3}+885.932572 \epsilon^{4}+O\left(\epsilon^{5}\right) .
\end{aligned}
$$

It transpires that the respective coefficients of the exponents are much larger than their $F_{4}$ counterparts. In effect what this means is that estimates for $E_{6}$ exponents from perturbation theory may only be reliable for a value of $d$ relatively close to six.

\section{Discussion.}

Our original aim was to extend the three loop analysis of $F_{4}$ symmetric scalar cubic theory in six dimensions to four loops. Having achieved this we derived critical exponents for the field and $\phi^{2}$-type operators in various representations of $F_{4}$ to the same order of precision. This is important in the context of predictions from the conformal bootstrap analysis of [25]. In that paper there was a suggestion that the difference in the $d=5$ dimension estimate for $\Delta_{\phi}$ compared to perturbative prediction was due to non-perturbative effects. From the Padé analysis we noted that the exponent derived from the $O\left(\epsilon^{4}\right)$ correction was smaller than the three loop result and, moreover, closer to that for the bootstrap. Although in $d=5$ there was not as sharp an estimate compared to the $d=5.95$ dimensional case. From the perturbative side the estimates for the dimension two operator exponents determined the order of their appearance in the spectrum. Close to six dimensions the two operators were effectively degenerate and the perturbative estimate for their exponents was in sharp agreement with [25]. For the lower dimensional case studied in [25] the value recorded there for $\Delta_{\mathbf{2 6}}^{2 \text { nd }}$ was consistent with the estimate for the $\phi^{2}$-type operator in the $\mathbf{3 2 4}$ representation rather than that in the $\mathbf{2 6}$ representation. It would be interesting to have a conformal bootstrap analysis for the exceptional group $E_{6}$. The observation of [25] that there appears to be a stable infrared fixed point in five dimensions seems to be confirmed. However, such a bootstrap analysis could give further insight into the role of the analogous $\phi^{2}$-type operator in $E_{6}$. From properties of the $E_{6}$ group it would appear that there is no operator parallel to that in the $\mathbf{3 2 4}$ representation of $F_{4}$. Instead there is only the $\mathbf{2 7}$ one. In other words a conformal bootstrap analysis should be able to estimate the $E_{6}$

value for what would be the exponent $\Delta_{\mathbf{2}}^{2 n}$ accurately and then compare with the four loop $\epsilon$ expansion. Equally the other groups in the $F_{4}$ family can be analysed by the bootstrap given that perturbative results are now available to the same accuracy as the $F_{4}$ case itself. 
Acknowledgements. The author thanks R.M. Simms for discussions. The work was carried out with the support of the STFC through the Consolidated Grant ST/L000431/1.

\section{A Exponents for related $F_{4}$ groups.}

While we have focused in the main on the exceptional group $F_{4}$ there are several other groups which have a tensor $d^{i j k}$ which satisfies the 4 -term relation of (3.2) and

$$
d^{i j j}=0 \quad, \quad d^{i k l} d^{j k l}=T_{2} \delta^{i j} .
$$

Using the Lie algebra classification notation these are $A_{1}, A_{2}$ and $C_{3}$, 25, 30, where $A_{1}$ is also equivalent to $B_{1}$. Values of the critical exponents for $\phi^{3}$ theory based on these symmetry groups can be deduced from the results of section 4 by setting $N=5,8$ and 14 respectively. Thus we have extended the three loop results of [25] for each case and found

$$
\begin{aligned}
\Delta_{\phi} & =\frac{1}{2} d-1+0.179487 \epsilon+0.174885 \epsilon^{2}+1.446636 \epsilon^{3}+11.125264 \epsilon^{4}+O\left(\epsilon^{5}\right) \\
\Delta_{\mathcal{O}^{(1)}} & =d-2-1.794872 \epsilon-2.641683 \epsilon^{2}-25.875476 \epsilon^{3}-179.737315 \epsilon^{4}+O\left(\epsilon^{5}\right) \\
\Delta_{\mathcal{O}^{(5)}} & =d-2+1.179487 \epsilon+0.174885 \epsilon^{2}+1.446636 \epsilon^{3}+11.125264 \epsilon^{4}+O\left(\epsilon^{5}\right) \\
\Delta_{\mathcal{O}^{(9)}} & =d-2-0.256410 \epsilon-1.214990 \epsilon^{2}-7.225638 \epsilon^{3}-54.804917 \epsilon^{4}+O\left(\epsilon^{5}\right) \\
\Delta_{\phi^{3}} & =d+2 \epsilon-3.880342 \epsilon^{2}-24.132914 \epsilon^{3}-250.285961 \epsilon^{4}+O\left(\epsilon^{5}\right)
\end{aligned}
$$

for $N=5$ corresponding to $A_{1}$. For $N=8$ which relates to the Lie algebra $A_{2}$ we have

$$
\begin{aligned}
\Delta_{\phi} & =\frac{1}{2} d-1+0.151515 \epsilon+0.041740 \epsilon^{2}+0.397533 \epsilon^{3}+2.024208 \epsilon^{4}+O\left(\epsilon^{5}\right) \\
\Delta_{\mathcal{O}^{(1)}} & =d-2-1.515152 \epsilon-0.959179 \epsilon^{2}-10.049808 \epsilon^{3}-38.922333 \epsilon^{4}+O\left(\epsilon^{5}\right) \\
\Delta_{\mathcal{O}^{(8)}} & =d-2+1.151515 \epsilon+0.041740 \epsilon^{2}+0.397533 \epsilon^{3}+2.024208 \epsilon^{4}+O\left(\epsilon^{5}\right) \\
\Delta_{\mathcal{O}^{(27)}} & =d-2-0.060606 \epsilon-0.521746 \epsilon^{2}-1.853121 \epsilon^{3}-9.180040 \epsilon^{4}+O\left(\epsilon^{5}\right) \\
\Delta_{\phi^{3}} & =d+2 \epsilon-2.389348 \epsilon^{2}-7.729109 \epsilon^{3}-65.712863 \epsilon^{4}+O\left(\epsilon^{5}\right) .
\end{aligned}
$$

We note that the sign of the $O\left(\epsilon^{3}\right)$ term of $\Delta_{\mathcal{O}^{(1)}}$ differs from that given in [25] which we assume is a typographical error. Finally, we find

$$
\begin{aligned}
\Delta_{\phi} & =\frac{1}{2} d-1+0.133333 \epsilon-0.011111 \epsilon^{2}+0.120049 \epsilon^{3}+0.437738 \epsilon^{4}+O\left(\epsilon^{5}\right) \\
\Delta_{\mathcal{O}^{(1)}} & =d-2-1.333333 \epsilon-0.244444 \epsilon^{2}-5.098686 \epsilon^{3}-10.110099 \epsilon^{4}+O\left(\epsilon^{5}\right) \\
\Delta_{\mathcal{O}^{(14)}} & =d-2+1.133333 \epsilon-0.011111 \epsilon^{2}+0.120049 \epsilon^{3}+0.437738 \epsilon^{4}+O\left(\epsilon^{5}\right) \\
\Delta_{\mathcal{O}^{(90)}} & =d-2+0.066667 \epsilon-0.260000 \epsilon^{2}-0.520808 \epsilon^{3}-1.816381 \epsilon^{4}+O\left(\epsilon^{5}\right) \\
\Delta_{\phi^{3}} & =d+2 \epsilon-1.611111 \epsilon^{2}-2.094961 \epsilon^{3}-20.962996 \epsilon^{4}+O\left(\epsilon^{5}\right)
\end{aligned}
$$

for $C_{3}$ having set $N=14$. The dimensions of $\mathcal{O}^{(R)}$ for $R \neq 1$ are determined from the respective $F_{4}$ expressions for the $\mathbf{2 6}$ and $\mathbf{3 2 4}$ representations which were computed as functions of $N$. Our expressions for $\Delta_{\phi}, \Delta_{\mathcal{O}^{(1)}}$ and $\Delta_{\phi^{3}}$ agree with the three loop ones given in the Appendix of [25], aside from the one noted above, but the four loop contributions are new. Also within our conventions the coefficients in the $\epsilon$ expansion of the $\phi^{2}$-type operators derived from the 26 representation of $F_{4}$ are in accord with $\Delta_{\phi}$ consistent with the relation to the conformal descendant operator. 


\section{References.}

[1] D. Friedan, Z. Qiu \& S.H. Shenker, Phys. Rev. Lett. 52 (1984), 1575.

[2] F.A. Dolan \& H. Osborn, Nucl. Phys. B599 (2001), 459.

[3] R. Rattazzi, V.S. Rychkov \& E. Tonni, JHEP 0812 (2008), 031.

[4] S. El-Showk, M.F. Paulos, D. Poland, S. Rychkov, D. Simmons-Duffin \& A. Vichi, Phys. Rev. D86 (2012), 025022.

[5] F. Kos, D. Poland \& D. Simmons-Duffin, JHEP 1406 (2014), 091.

[6] G. Mack \& A. Salam, Annals Phys. 53 (1969), 174.

[7] S. Ferrara, A.F. Grillo \& R. Gatto, Lett. Nuovo Cim. 2S2 (1971), 1363.

[8] S. Ferrara, A.F. Grillo, G. Parisi \& R. Gatto, Nucl. Phys. B49 (1972), 77.

[9] S. Ferrara, A.F. Grillo \& R. Gatto, Annals Phys. 76 (1973), 161.

[10] S. Ferrara, A.F. Grillo, R. Gatto \& G. Parisi, Nuovo Cim. A19 (1974), 667.

[11] S. Ferrara, A.F. Grillo \& R. Gatto, Phys. Rev. D9 (1974), 3564.

[12] A.M. Polyakov, Zh. Eksp. Teor. Fiz. 66 (1974), 23.

[13] A. Pelissetto \& E. Vicari, Phys. Rept. 368 (2002), 549.

[14] L. Fei, S. Giombi \& I.R. Klebanov, Phys. Rev. D90 (2014), 025018.

[15] L. Fei, S. Giombi, I.R. Klebanov \& G. Tarnopolsky, Phys. Rev. D91 (2015), 045011.

[16] J.-B. Bae \& S.-J. Rey, arXiv:1412.6549 [hep-th].

[17] S.M. Chester, S.S. Pufu \& R. Yacoby, Phys. Rev. D91 (2015), 086014.

[18] F. Kos, D. Poland, D. Simmons-Duffin \& A. Vichi, JHEP 1511 (2015), 106.

[19] P. Mati, Phys. Rev. D94 (2016), 065025.

[20] A. Eichhorn, L. Janssen \& M.M. Scherer, Phys. Rev. D93 (2016), 125021.

[21] K. Kamikado \& T. Kanazawa, arXiv:1604.04830 [hep-th].

[22] Z. Li \& N. Su, arXiv:1607.07077 [hep-th].

[23] T. Banks \& A. Zaks, Nucl. Phys. B196 (1982), 189.

[24] J.A. Gracey, Phys. Rev. D92 (2015), 025012.

[25] Y. Pang, J. Rong \& N. Su, JHEP 1612 (2016), 057.

[26] O.F. de Alcantara Bonfim, J.E. Kirkham \& A.J. McKane, J. Phys. A13 (1980), L247; A13 (1980), 3785.

[27] O.F. de Alcantara Bonfim, J.E. Kirkham \& A.J. McKane, J. Phys. A14 (1981), 2391.

[28] A.L. Pismensky, Int. J. Mod. Phys. A30 (2015), 1550138. 
[29] A.N. Vasil'ev, Y.M. Pismak \& J.R. Honkonen, Theor. Math. Phys. 50 (1982), 127.

[30] P. Cvitanovic, Group theory: Birdtracks, Lie's and exceptional groups, (Princeton University Press, Princeton, USA, 2008).

[31] P. Nogueira, J. Comput. Phys. 105 (1993), 279.

[32] J.A.M. Vermaseren, math-ph/0010025.

[33] M. Tentyukov \& J.A.M. Vermaseren, Comput. Phys. Commun. 181 (2010), 1419.

[34] S. Laporta, Int. J. Mod. Phys. A15 (2000), 5087.

[35] C. Studerus, Comput. Phys. Commun. 181 (2010), 1293.

[36] A. von Manteuffel \& C. Studerus, arXiv:1201.4330 [hep-ph].

[37] A.J. McKane, D.J. Wallace \& R.K.P. Zia, Phys. Lett. B65 (1976), 171.

[38] A.J. McKane, J. Phys. G3 (1977), 1165.

[39] T.W. Kephart \& M.T. Vaughn, Z. Phys. C10 (1981), 267.

[40] H. Freudenthal, Adv. Math. 1 (1964), 145.

[41] H. Freudenthal, Proc. Kon. Ned. Akad. Wet. A57 (1954), 218.

[42] H. Freudenthal, Proc. Kon. Ned. Akad. Wet. A62 (1959), 447.

[43] T.A. Springer, Proc. Kon. Ned. Akad. Wet. A65 (1962), 259.

[44] P. Cvitanovic, Phys. Rev. D14 (1976), 1536. 